\title{
IMPORTÂNCIA DA TEMÁTICA TUBERCULOSE NA GRADUAÇÃO EM ENFERMAGEM: A DISCURSIVIDADE DOS DOCENTES
}

\author{
THE IMPORTANCE OF TUBERCULOSIS \\ AS A THEME IN NURSING GRADUATION: \\ THE DISCOURSE OF PROFESSORS
}

\section{LA IMPORTANCIA DEL TEMA TUBERCULOSIS EN LA GRADUACIÓN EN ENFERMERÍA: EL DISCURSO DE LOS PROFESORES}

\author{
Rosana Santos Brandão de Assis ${ }^{1}$ \\ Lara Bezerra de Oliveira ${ }^{2}$ \\ Luana Cristina Fernandes Eufrasio ${ }^{3}$ \\ Ana Paula Carvalho Portela ${ }^{4}$ \\ Pedro Fredemir Palha ${ }^{5}$ \\ Amélia Nunes Sicsú ${ }^{6}$
}

Como citar este artigo: Assis RSB, Oliveira LB, Eufrasio LCF, Portela APC, Palha PF, Sicsú AN. Importância da temática tuberculose na graduação em enfermagem: a discursividade dos docentes. Rev baiana enferm. 2019:33:e30472

Objetivo: analisar a discursividade dos docentes sobre a importância da temática tuberculose nos cursos de graduação em Enfermagem. Método: estudo qualitativo do tipo exploratório, desenvolvido entre janeiro e março de 2015 em duas universidades públicas de Enfermagem do estado do Amazonas, Brasil. Participaram do estudo 15 docentes do curso de Enfermagem, sendo a maioria do sexo feminino, possuía mestrado, com mais de cinco anos na docência, experiência anterior à docência e apenas um vínculo profissional. Utilizou-se a Análise do Discurso de matriz francesa como princípio teórico-metodológico. Resultados: emergiram, como resultados, dois blocos discursivos: Importância da temática da tuberculose na estrutura curricular; e Papel do enfermeiro no controle da tuberculose. Conclusão: os sentidos produzidos pelo discurso dos docentes permitiram concluir que a tuberculose, por ser uma doença importante do ponto de vista epidemiológico e social, precisa ter maior abrangência na matriz curricular dos cursos de graduação em Enfermagem, tendo vista o protagonismo dessa profissão nas ações de controle da doença.

Descritores: Tuberculose. Enfermagem. Docentes de Enfermagem. Ensino Superior. Instituições de Ensino Superior.

Objective: analyzing the discourses of professors about the importance of the theme tuberculosis in Nursing graduation courses. Method: qualitative, exploratory study, developed from January to March 2015 in two public Nursing

\footnotetext{
Enfermeira. Pesquisadora independente. Manaus, Amazonas, Brasil. https://orcid.org/0000-0002-445I-7066

Enfermeira. Especialista em Enfermagem em Terapia Intensiva. Universidade do Estado do Amazonas. Manaus, Amazonas, Brasil. larabezerradeassis@gmail.com. https://orcid.org/0000-000 I-9565-7088

3 Estudante de Enfermagem. Universidade do Estado do Amazonas. Manaus, Amazonas, Brasil. https://orcid.org/0000-0002-| 843-8364

4 Enfermeira. Mestre em Condições de Vida e Situação de Saúde na Amazônia. Professora da Universidade do Estado do Amazonas. Manaus, Amazonas, Brasil. https:// orcid.org/0000-0002-2594-4708

5 Enfermeiro e Obstetra. Doutor em Enfermagem em Saúde Pública. Professor da Universidade de São Paulo. Ribeirão Preto, São Paulo, Brasil. https://orcid.org/00000002-5220-4529

6 Enfermeira. Doutora em Ciências. Professora da Escola Superior de Ciências da Saúde. Manaus, Amazonas, Brasil. https://orcid.org/0000-000 I-52 17-37I0
} 
universities in the state of Amazonas, Brazil. Fifteen professors from the Nursing course participated in the study. Most were female, had master's degrees, were teachers for more than five years, had working experience prior to being professors, and only one job. The study used Discourse Analysis from a French theoretical-methodological framework. Results: two discursive blocks emerged as results: The Importance of the Theme Tuberculosis in the Syllabus; and The Role of the Nurse in Tuberculosis Control. Conclusion: the meanings produced by the discourses of the professors made it possible to conclude that, since tuberculosis is an important disease from an epidemiological standpoint, it must be more broadly present in the syllabus of Nursing graduation courses, due to the main role these professionals have in the actions to control this disease.

Descriptors: Tuberculosis. Nursing. Faculty, Nursing. Education, Higher. Higher Education Institutions.

Objetivo: analizar los discursos de profesores sobre la importancia del tema tuberculosis en los cursos de graduación en Enfermería. Método: estudio exploratorio, cualitativo, desarrollado de enero a marzo de 2015 en dos universidades de Enfermería en el estado de Amazonas, en Brasil. Quince docentes del corso de Enfermería participaron del estudio. La mayoría eran mujeres, con másteres, profesoras hace más de cinco años, con experiencias de trabajo antes que fueran profesoras, y solamente un trabajo. El estudio utilizó el Análisis de Discurso de base teórica y metodológica francesa. Resultados: dos blocos discursivos emergieron como resultados: La importancia del tema tuberculosis en el programa del corso; y El papel de los enfermeros en el control de tuberculosis. Conclusión: los significados producidos por los discursos de los profesores posibilitaron concluir que, puesto que la tuberculosis es una enfermedad importante desde un punto de vista epidemiológico, necesita estar más presente en los programas de cursos de graduación en Enfermería, gracias al papel esencial de esos profesionales en las acciones de control de esa enfermedad.

Descriptores: Tuberculosis. Enfermería. Docentes de Enfermería. Educación Superior. Instituciones de Enseñanza Superior.

\section{Introdução}

A tuberculose (TB) permanece como principal causa de morbimortalidade no mundo, apesar dos avanços no tratamento quimioterápico e de técnicas diagnósticas mais eficientes, sendo responsável por cerca de 9,6 milhões de casos novos e 1,5 milhões de mortes anualmente $^{(1)}$. É considerada um importante problema de saúde pública mundial ${ }^{(2)}$.

O estado do Amazonas destaca-se, por registrar o maior número de casos de TB em todo o território nacional, apresentando coeficientes de incidência da doença (70,1/100.000 hab.), mortalidade (3,3/100.000 hab.) e abandono de tratamento (13,3/100.000 hab.) maiores que as taxas nacionais ${ }^{(3)}$.

Nesse cenário, incontáveis estudos são realizados mundialmente, objetivando contribuir para redução do número de casos e para o tão esperado controle da doença. Todavia, são muitos os obstáculos enfrentados pelos programas de controle da TB que dificultam o alcance das metas almejadas. Esses vão desde as questões políticas até as questões operacionais da equipe de saúde ${ }^{(1-4)}$.
Desde o ressurgimento da $\mathrm{TB}$, há quase três décadas, tem ocorrido uma crescente preocupação com a educação e a competência dos profissionais de saúde para lidar com questões especializadas inerentes a essa doença ${ }^{(4)}$. O conhecimento sobre a TB é essencial para os universitários da área de Enfermagem, pois são atores importantes na efetivação do cuidado dessa doença, na medida em que gerenciam as ações de controle e compreendem a complexidade envolvida nesse processo ${ }^{(5)}$. Desse modo, é necessário o desenvolvimento da consciência crítica sobre as próprias atitudes, tanto referente às ações que visam a sua autoproteção, como à do paciente, precavendo-se, inclusive, das atitudes antiéticas, não humanísticas, estigmatizadoras e preconceituosas.

Nesse sentido, enfatiza-se a importância das instituições de ensino superior, como espaços potencializados de difusão do conhecimento geral e não somente específico; formação de profissionais em conhecimentos, valores e formas de pensar e agir, bem como responsável por criar mudanças que implicam na quebra de 
paradigmas $^{(6)}$. É nesse cenário que figura o profissional docente como um importante elemento que contribui para o despertar das potencialidades do estudante, propiciando espaços para a construção do conhecimento ${ }^{(7)}$.

Em breve levantamento bibliográfico nas bases de dados Lilacs, Pubmed, Web of Science, sobre a percepção de docentes em relação à inserção da temática de TB nos cursos de graduação em Enfermagem, não foram encontrados estudos nessa perspectiva tanto no cenário nacional quanto internacional. Assim, diante da relevância do papel docente na formação dos estudantes e da importância epidemiológica da TB em cenário mundial e, sobretudo, no estado do Amazonas, torna-se importante compreender o discurso dos docentes sobre a inserção dessa temática nos cursos de graduação em Enfermagem.

Frente ao exposto, este estudo teve como objetivo analisar a discursividade dos docentes sobre a importância da temática tuberculose nos cursos de graduação em Enfermagem.

\section{Método}

Este é um estudo qualitativo, do tipo exploratório, que utilizou a Análise do Discurso (AD), de matriz francesa, como princípio teórico-metodológico para compreender os sentidos produzidos pelos discursos dos docentes sobre a importância da temática da TB nos cursos de graduação em Enfermagem.

O estudo foi aprovado pelo Comitê de Ética em Pesquisa da Universidade do Estado do Amazonas, CAAE: 26192114000005016, e atendeu às normas éticas e legais contidas na Resolução n. 466/12 do Conselho Nacional de Saúde ${ }^{(8)}$.

Para a AD de matriz francesa, que tem Michel Pêcheux como fundador, confluem três grandes áreas do conhecimento: Psicanálise, Linguística e Materialismo histórico. A Linguística explica os mecanismos sintáticos e os processos de enunciação; a Psicanálise, a subjetividade e a relação do sujeito com o simbólico; e o Materialismo histórico, os fenômenos das formações sociais, incluindo a ideologia que se manifesta no discurso enquanto efeito de sentido entre os interlocutores, o qual pertence a um campo de contradições e estranhamentos ${ }^{(9)}$.

A AD surgiu na década de 60 do século XX e tem como objeto próprio o discurso: a língua funcionando para a produção de sentidos. Por meio do discurso, acredita-se na possibilidade de acessar o que há de singular no sujeito, isto é, o inconsciente, assim como as marcas sociais e históricas que o caracterizam ${ }^{(10)}$.

Para melhor compreensão do referencial e da análise dos dados, é necessário refletir sobre conceitos clássicos na AD, como: sujeito, ideologia, condições de produção, formação discursiva e interdiscurso ${ }^{(9)}$.

Entende-se por sujeito, as posições que um determinado indivíduo ocupa enquanto um enunciador interpelado pela ideologia, ou seja é um sujeito determinado pela língua e pela história. Nesse contexto, trata-se de um sujeito clivado. Não há discurso sem sujeito e sujeito sem ideologia. A ideologia é a interpretação de sentido em uma direção determinada pela relação da linguagem com a história. As condições de produção compreendem fundamentalmente os sujeitos e a situação, além da memória, que também faz parte da produção do discurso ${ }^{(9)}$. As condições de produção precisam ser levadas em consideração, para que a análise explique o funcionamento discursivo, apontando uma memória discursiva, em que as contradições entre as formações discursivas são facilmente percebidas. A formação discursiva é concebida como aquilo que, numa formação ideológica dada, isto é, de uma posição dada numa conjuntura dada, determina o que pode e deve ser dito ${ }^{(9)}$. À luz desse pensamento, percebe-se que o discurso sempre estará vinculado a outros discursos, o interdiscurso, compreendido como "[...] aquilo que fala antes, em outro lugar, independentemente" ${ }^{(9: 10)}$.

O estudo foi desenvolvido em Manaus, estado do Amazonas, em duas universidades públicas de Enfermagem comprometidas com o ensino, a pesquisa e a extensão.

Participaram do estudo 15 docentes do curso de Enfermagem. A maioria era do sexo feminino (10), possuía mestrado (9), com mais de 5 anos 
na docência (9), e apenas um vínculo profissional, no caso como docente (11), e com experiência anterior à docência (12) em hospital (5), na Atenção Primária à Saúde (4), em urgência e emergência (3), na gerência (1), em Unidade de Terapia Intensiva (1), centro cirúrgico (1) e vigilância epidemiológica (1). Constituíram-se critérios de inclusão: ministrar conteúdos relacionados à $\mathrm{TB}$ e estar em atividade docente no período da coleta de dados.

As entrevistas foram realizadas entre os meses de janeiro e março de 2015 em local indicado pelo docente. Teve duração média de 15 minutos, sendo registrada em um gravador digital após assinatura do Termo de Consentimento Livre e Esclarecido pelos participantes. A entrevista foi norteada pela seguinte frase disparadora do discurso: Comente sobre a importância da temática tuberculose na matriz curricular do curso de graduação em Enfermagem.

As entrevistas foram audiogravadas, transcritas na íntegra e organizadas no Excel, onde a coluna correspondeu à questão norteadora e as linhas, aos participantes. Cada linha do Excel correspondeu a um documento primário (Primary Documents). Em seguida, esses dados foram exportados para o software Atlas.ti 7.0 e criado um único projeto denominado "Unidade Hermenêutica (Hermeneutic Unit)". Esclarece-se que o software não interferiu na escolha dos recortes, nem na criação dos blocos; apenas auxiliou na organização dos dados cuja seleção e comando foram realizados exclusivamente pelos pesquisadores. Na apresentação dos resultados, foi utilizada a letra D para docente, seguida do número correspondente à sequência das entrevistas.

Para análise das entrevistas, utilizou-se a AD seguindo três etapas: passagem da superfície linguística para o objeto discursivo - realizaram-se leituras das entrevistas transcritas, observando a discursividade e identificando as enunciações indiciadoras para a interpretação, desnaturalizando-se, dessa forma, a palavra das coisas; do objeto discursivo para o processo discursivo - identificaram-se as sequências discursivas, relacionando-as com as distintas formações discursivas, delineando os sentidos circulantes nos fragmentos em análise; processo discursivo em si (formação ideológica) - interpretação das Sequências Discursivas (SD), observando as condições de produção e as formações discursivas nas quais os sujeitos inscrevem-se e o que fundamenta os enunciados ${ }^{(9)}$.

\section{Resultados}

Do discurso dos sujeitos docentes, emergiram dois blocos discursivos: Importância da temática da tuberculose na matriz curricular e Papel do enfermeiro nas ações de controle da tuberculose.

\section{Importância da temática da tuberculose na matriz curricular}

Neste bloco, emergem discursos que se inscrevem em formações discursivas que enfatizam a TB como uma doença endêmica, reemergente, negligenciada e socialmente constituída.

Os dizeres dos sujeitos docentes D1, D9 e D14 estão inscritos em um discurso epidemiológico que enfatiza a alta incidência de TB no Amazonas. Além disso, sinalizam a importância do conhecimento adquirido sobre a TB durante a graduação para a futura prática profissional, como pode depreender-se dos recortes a seguir: Porque o acadêmico que vai ser o futuro enfermeiro deve
conhecer a doença específica da região, além de ser de
alta incidência na nossa realidade. (D1).

O Amazonas é campeão de número de casos notificados de tuberculose. Com o objetivo de desmistificar a TB [tuberculose] e induzir ao diagnóstico precoce, é importante o tema tuberculose nos cursos de graduação em Enfermagem. (D9).

Por ser uma doença com elevada incidência no Brasil, com implicações sociais. Igualmente, por ser uma doença endêmica no estado do Amazonas, é fundamental que o aluno tenha conbecimento sobre a prevenção da doença, pois o controle da doença é o ponto mais importante no processo de diminuição dos casos em nosso país. (D14).

O papel da universidade, por intermédio dos docentes, no controle de doenças endêmicas na região, como a TB, pode ser percebido no recorte seguinte:

[...] e nós, da enfermagem, temos esse compromisso. Se você vive numa região que tem o maior número de casos de tuberculose, e o enfermeiro sai do curso sem saber lidar com isso, é bastante complexo. Então, assim, nós, formadores, professores, temos a obrigação de formar o aluno sabendo disso, de como controlar, como combater e como prevenir a TB. (D13). 
O conhecimento sobre TB é apontado como uma forma de empoderamento dos estudantes para as ações de controle da doença, conforme apresentado no recorte a seguir:

[...] o aluno já deve sair preparado para o mercado de trabalho com conbecimento prévio do que fazer. Quanto mais conbecimento o aluno tiver, mais rápido vai conseguir fazer um diagnóstico e minimizar os danos tanto para o paciente quanto para os demais contactantes, seja familiar ou profissional dentro da rede social dele. (D4).

A TB, como uma doença reemergente, surge como ponto basilar nos recortes dos sujeitos docentes D2 e D5.

Porque é uma doença [...] considerada como uma doença reemergente, que já tinha sido notificada há muito tempo e há algum tempo foi considerada como controlada, ou seja, quase erradicada, e boje em dia ela está sendo considerada como um dos principais problemas de saúde pública. Em alguns estudos, isso é associada [à] emergência do HIV, ou seja, emergência, porque é uma doença nova que entra na sociedade, e reemergente, porque é uma doença que já existia, mas que estava controlada e agora volta de novo a preocupar a saúde pública na população. Em alguns estudos, se diz que essa reemergência da TB se dá com a associação do HIV-Aids. Ela deve ser considerada e deve ser tratada com muita responsabilidade. Ela é, de fato, uma doença que está vindo com muita força. (D2).

[...] porque a tuberculose reemergente, nos últimos 10 anos, concomitante com HIV, é é importante que o aluno saiba lidar com esse paciente. (D5).

Os discursos dos sujeitos docentes D4 e D11 fazem parte de uma formação discursiva que percebe a TB como uma doença negligenciada, como pode depreender-se nos recortes a seguir:

[...] é uma doença negligenciada. É uma doença infectocontagiosa, que precisa mesmo de uma atenção especial de políticas públicas voltadas pra prevenção, proteção. E os futuros profissionais precisam ter conbecimento de como trabalhar as questões públicas. (D4).

[...] por estar inclusa como Doença Negligenciada e de notificação compulsória, que requer do enfermeiro conhecimentos científicos sobre a doença, especialmente sua sintomatologia, para que o enfermeiro possa sistematizar o cuidado de enfermagem. A profundidade de conbecimento conduzirá o enfermeiro desde a promoção da saúde da população ao tratamento do ser doente de TB. (D11).

Ainda neste bloco discursivo, os dizeres seguintes enunciam o caráter social que permeia a TB:

A TB é uma doença socialmente constituída e, além de ser uma doença infectocontagiosa, o local onde a pessoa vive, as condições de vida, é importantíssimo; a primeira coisa que o aluno tem que saber é isso. Qual é o contexto social em que o indivíduo está inserido na sociedade e onde ele, como futuro enfermeiro, irá trabalbar? A partir do momento em que eu sei qual é o local em que as pessoas então inseridas, eu vou fazer o que? Trabalhar melhor a prevenção e como prevenir, fazendo busca ativa dos sintomáticos respiratórios, no caso da TB pulmonar, o mais precocemente possivel, porque, às vezes, como a gente sabe que a TB é uma doença que quase todo mundo tem o bacilo, o que vai manifestar é a condição que o paciente está envolvido. Em questão da tuberculose, é fundamental o aluno entender tudo isso, pra poder trabalhar a ideia da prevenção. (D13).

Sim, porque é uma doença que atinge a população com maior vulnerabilidade socioeconômica e ainda é um problema nos dias atuais. (D15).

\section{Papel do enfermeiro nas ações de controle da tuberculose}

As posições-sujeito afetadas pela ideologia, nas condições de produção existentes, ao discursarem inscrevem-se numa formação discursiva que compreende o protagonismo do enfermeiro nas ações de controle da TB como importante. Nesse contexto, é indicado como importante o conhecimento dos estudantes sobre a temática da $\mathrm{TB}$, pelo fato de poderem ser profissionais aplicadores do conhecimento adquirido, organizadores, responsáveis ou gestores das unidades sanitárias, conforme se pode compreender no material simbólico presente nos recortes que seguem:

[...] é importante que os alunos finalistas saibam, porque vão ser os futuros enfermeiros e serão linha de frente em uma unidade hospitalar. Querendo ou não, o enfermeiro é porta de entrada. (D3).

Os alunos serão futuros enfermeiros que estarão na linha de frente nos programas de saúde governamentais voltados para TB. (D12).

O enfermeiro é um dos principais profissionais na organização e andamento do programa de TB. (D14).

É importante porque os finalistas logo estarão ocupando seus espaços nos diversos níveis e modelos de atenção à saúde e principalmente aqueles que atuarão na atenção primária à saúde, terão um papel fundamental para a realização de ações primordiais nesse nível, tal como busca ativa de sintomáticos respiratórios, diagnóstico e tratamento, além das ações de educação em saúde junto à comunidade. (D8).

[...] é importante abordar na grade curricular, para o então discente/aluno já ter uma noção desse conbecimento e aplicar na prática [...] porque o enfermeiro, no caso discente, quando formar, já ter esse conbecimento e aplicar em assistência. Na saúde pública, ele coordena, ele aplica diretamente os cadernos dessas doenças, de acordo com as orientações do Ministério da Saúde, fazendo diagnóstico, prescrevendo ou transcrevendo medicações, entre outros cuidados. Por isso, é importante. (D6). 
A necessidade de uma abordagem multidisciplinar para o controle da doença é enunciada no recorte que segue:

Devido à TB ser um agravo que necessita de intervenção de equipe multiprofissional de assistência a esses indivíduos portadores da doença e inseridos nesta equipe, o enfermeiro [...] o aluno deve estar preparado sobre a abordagem profissional sobre a prevenção, o diagnóstico precoce da doença [constitui ferramenta importante no controle da doençal, sobre as formas de tratamento $e$ cuidado ao paciente e o preparo no atendimento, consolidando a possibilidade do ciclo de transmissão da doença, por meio da identificação precoce dos fatores determinantes e condicionantes da doença e que contribuem para o aumento das taxas de incidência. (D7).

\section{Discussão}

No primeiro bloco discursivo, "Importância da temática da TB na matriz curricular", verifica-se, nas entrelinhas dos recortes 1, 2 e 3, em que os sujeitos docentes apontam a necessidade de expandir a temática da TB na matriz curricular, por ser uma doença "específica da região", o estado ser "o campeão de número de casos notificados de tuberculose", e ser "uma doença endêmica", o estudante irá deparar-se com situações envolvendo a doença em sua futura prática profissional. O fato de a TB ter uma alta incidência no estado do Amazonas não significa que é específica da região. A TB é uma doença de magnitude mundial, como bem mencionado na literatura, em que 22 países, entre eles o Brasil, possuem cerca de $80 \%$ dos casos da doença ${ }^{(11)}$. O significante "campeão" traz um paradigma indiciário, que instiga a pensar que o estado do Amazonas é o mais afetado pela doença. Como já mencionado, o estado está em primeiro lugar no Brasil em número de casos de $\mathrm{TB}^{(3)}$.

A sequência discursiva do sujeito D9 "desmistificar a TB" produz sentidos que reforçam o sentido negativo da causa da doença e das suas formas de transmissão constituídos historicamente no imaginário social dos docentes, dos estudantes e da população. A literatura aponta que a TB é considerada uma doença metafórica, despertando um imaginário alimentado por explicações fantasiosas e estigmatizantes em relação à doença, as quais trazem consequências biológicas e sociais para a pessoa portadora ${ }^{(12)}$.
O estigma é um dos principais obstáculos para o controle da TB e pode retardar a procura da pessoa com a doença pelos serviços. Por isso, é importante que os profissionais de saúde desenvolvam qualidades como empatia, preocupação e respeito com a pessoa com $\mathrm{TB}$, além de sensibilidade cultural $^{(13)}$.

É notável, nos discursos dos docentes, a importância da temática da TB. Eles referem a importância do conhecimento dos estudantes sobre a doença e os resultados positivos para o controle da doença, se o ensino estiver comprometido a cooperar na formação de estudantes em relação a uma doença tão presente no estado. No entanto, estudo recente realizado com estudantes sobre o conhecimento sobre a TB, que mostrou a vulnerabilidade em relação ao grau de conhecimento sobre a doença, possibilita a reflexão sobre as metodologias utilizadas na formação e qualificação dos futuros enfermeiros, para possibilitar integrar diferentes tipos de conhecimento para uma atuação profissional qualificada no cuidado às pessoas com $\mathrm{TB}^{(5)}$.

Ainda no primeiro bloco, observa-se que o docente D13, tomando a posição de formador de opiniões, assume a corresponsabilidade de contribuir para o controle da TB. Destaca-se que as Instituições de Ensino Superior devem ser comprometidas com a formação de profissionais qualificados a responder pelas demandas do Sistema Único de Saúde (SUS). Devem também cumprir seus princípios, como equidade, universalidade e integralidade ${ }^{(14)}$, destacando a importância do SUS no controle da TB, uma vez que possibilita o acesso gratuito ao diagnóstico e ao tratamento dessa doença.

A sequência discursiva "o aluno já deve sair preparado para o mercado de trabalho, com conhecimento prévio do que fazer" (D4), parece incorporar um discurso institucional que enfatiza indiretamente o papel da universidade na formação e preparação de profissionais aptos para serem inseridos no mercado de trabalho com as habilidades e competências necessárias para desempenharem sua função. O modelo de saúde hodierno exige qualificação profissional para o atendimento das necessidades da população e 
dos serviços de saúde. Assim, as atividades de educação permanente devem estar direcionadas às necessidades de saúde da população, sendo indispensáveis profissionais com competências e habilidades para atuarem de forma consciente e reflexiva ante as reais necessidades e prioridades de saúde da população ${ }^{(15)}$.

A sequência discursiva "Quanto mais conhecimento o aluno tiver mais rápido vai conseguir fazer um diagnóstico“ (D4) sinaliza um dizer que aponta o conhecimento sobre a doença como imprescindivel para um diagnóstico precoce. Considera-se que o conhecimento teórico e científico sobre TB é fundamental para os estudantes da área de saúde, pois ocuparão importantes funções nas ações de controle da doença. Destaca-se que, ao enfermeiro, cabe a atuação direta junto às pessoas com TB, seja na prevenção, seja na promoção de saúde, de forma individualizada ou coletiva. Ressalta-se que a prevenção e a educação em saúde constituem-se em meios inquestionáveis para evitar o surgimento de novos casos, sendo também veículo de conscientização e informação. Assim, é importante apropriarem-se desses conhecimentos para, com base neles, estimularem o empoderamento da pessoa com TB como instrumento de transformação social, consciente, capaz de garantir mudanças de comportamento e atitudes ${ }^{(5,16)}$.

Uma memória histórica ecoa e reverbera no discurso do sujeito D2, quando enuncia que a TB é uma doença reemergente, que recrudesceu com o advento da Aids. Observa-se que o significante "reemergente" está presente nos discursos dos docentes e também de muitos pesquisadores da área, todavia concorda-se que a TB pode ser reemergente para alguns países europeus, e mesmo para os Estados Unidos da América, mas, no Brasil, a TB não é problema de saúde pública emergente e tampouco reemergente ${ }^{(17)}$. Ela é um problema presente e permanente há longo tempo, muitas vezes negligenciado.

Os sujeitos D4 e D11, afetados pelo interdiscurso, ao enunciarem as sequências discursivas "é uma doença negligenciada", "é uma doença [...] que precisa mesmo de uma atenção especial de políticas públicas [...]" e "está inclusa como doença negligenciada" incorporam e reafirmam o interdiscurso de que a TB é uma doença negligenciada e esquecida das políticas públicas $^{(17)}$. Ainda nesses recortes, circulam novamente os sentidos sobre a necessidade de o acadêmico e futuro enfermeiro possuir conhecimentos sobre a TB.

Nos dizeres do sujeito D13, os efeitos de sentidos de que a TB é uma doença socialmente constituída são reverberados mediante a memória discursiva. Sinaliza-se ainda, nesse recorte, um imaginário representado na sequência discursiva "o local onde a pessoa vive, as condições de vida é importantíssimo; a primeira coisa que o estudante tem que saber é isso”. Esse imaginário está ancorado em uma formação discursiva que compreende a importância de os estudantes de enfermagem conhecerem o contexto social em que está inserida a pessoa com TB e atuarem de forma humana e integral, pois assim é possível identificar as necessidades dessa pessoa e propor estratégias de atuação tangíveis. Vale ressaltar que a formação de profissionais enfermeiros deve estar voltada para a integralidade dos problemas de saúde da população brasileira, necessitando ser contextualizada e resolutiva, fundamentando-se em Projetos Pedagógicos de Cursos que possibilitem práticas de saúde com responsabilidade social ${ }^{(18)}$.

O sujeito D15 destaca a vulnerabilidade social e econômica que acomete os sujeitos adoecidos por TB. A sinergia entre TB e pobreza é fato respaldado na literatura. Ela é historicamente descrita como uma doença social, que se desenvolve num contexto de pobreza e desvantagem social ${ }^{(19-21)}$. Destacam-se não somente os fatores relacionados à pobreza, que aumentam a probabilidade de infecção, como também as despesas relacionadas à doença e a perda nos rendimentos que impedem o cuidado, aprofundam o empobrecimento e aumentam o risco de reações adversas e recorrência ${ }^{(21)}$.

No segundo bloco discursivo "Papel do enfermeiro nas ações de controle da Tuberculose", as sequências discursivas "em uma unidade hospitalar", "nos programas de saúde governamentais voltados para TB", "andamento do programa 
de TB" e "ocupando espaços nos diversos níveis e modelos de atenção à saúde" indiciam as diferentes posições que o enfermeiro ocupa no exercício de sua profissão e que podem estar relacionadas com as ações de controle da TB.

A metáfora "linha de frente", presente no discurso dos sujeitos D3 e D12, no interdiscurso, refere-se ao local para onde os generais mandavam seus melhores soldados, produzindo, assim, no discurso dos docentes, o sentido do papel protagonista do enfermeiro nos serviços de saúde. A Enfermagem tem se mostrado detentora de um discurso legítimo sobre as questões relacionadas à assistência às pessoas com TB, com grande experiência do controle da doença, tanto no ambiente hospitalar quanto na saúde pública, envolvendo a prevenção, o tratamento e a formação de trabalhadores de saúde para atuação frente à doença ${ }^{(16,22)}$.

A sequência discursiva "os alunos serão futuros enfermeiros" impele a cogitar que o ser enfermeiro é um sujeito assujeitado na organização e gestão do programa da TB: "O enfermeiro é um dos principais [...] na organização e andamento do programa de TB" (D14). Outrossim os mesmos dizeres produzem sentidos que instigam a pensar que, embora os significantes "futuros enfermeiros" estejam presentes no discurso dos docentes, o discente já se depara, no decorrer do percurso acadêmico e durante seus estágios, com situações que envolvem a pessoa com TB. Por isso, esse tema deve ser debatido em diversos momentos de sua formação. Por outro lado, esses significantes servem como um chamamento, para que o estudante compreenda a sua responsabilidade nas ações de saúde como futuro profissional.

A sequência discursiva "terão um papel fundamental para a realização de ações primordiais: busca ativa de sintomáticos respiratório [...]" (D8) permitiu observar-se um sujeito afetado pelo interdiscurso e interpelado pela ideologia, inscrevendo-se em formação discursiva do discurso médico ancorado em dizeres do programa brasileiro de controle da TB e da OMS, que entendem a busca ativa de sintomáticos como um dos processos importantes no controle da doença ${ }^{(11,23)}$.
Os significantes "devido a TB ser um agravo que necessita de intervenção de equipe multiprofissional", por um lado produzem o sentido de que a assistência à pessoa com TB envolve a intervenção de vários profissionais e, nesse sentido, o ensino que enfoque a atuação multidisciplinar é importante no controle da moléstia. Por outro lado, esses mesmos dizeres deixam vestígios que impelem a refletir que a figura do enfermeiro é instigada pelo complexo processo de gestão e atuação em equipe. A literatura tem apontado a extrema relevância da presença de uma equipe completa, podendo ser um fator influente na redução do abandono do tratamento. Por isso, é importante que o Programa de Controle da Tuberculose priorize estratégias desenvolvidas por equipes multiprofissionais, objetivando a inclusão social do paciente e reforçando a organização do serviço ${ }^{(24)}$.

O discurso educacional-autoritário "o aluno deve estar preparado sobre a abordagem profissional, sobre a prevenção, o diagnóstico precoce da doença" possibilitou sinalizar-se a condição sine qua non que determina o reconhecimento do estudante como profissional competente e aceito pela classe. O conhecimento sobre a prevenção e o diagnóstico das moléstias constituem elementos básicos para o futuro enfermeiro, visto que a falta desse pode tornar deficiente $\mathrm{O}$ cuidado à pessoa com TB e contactantes, o que potencializa o risco de transmissão da doença entre os profissionais e na população ${ }^{(25)}$.

Este estudo teve como possível limitação o fato de não permitir verificar se, na prática docente dos profissionais entrevistados, há maior preocupação em inserir o tema da $\mathrm{TB}$, todavia esta indagação é propulsora para outros estudos.

\section{Conclusão}

Os sentidos produzidos pelo discurso dos profissionais permitiram concluir que a TB é uma doença importante do ponto de vista epidemiológico e social e precisa ter maior abrangência na matriz curricular dos cursos de graduação em Enfermagem, tendo vista o protagonismo dessa profissão nas ações de controle da doença. 
O processo de ensino-aprendizagem sobre a TB precisa ser ressignificado constantemente pelos docentes e pela instituição de ensino superior, para que o perfil do egresso seja o de um profissional capaz de atuar nas atividades de controle dessa doença de grande relevância epidemiológica.

\section{Fontes de Financiamento:}

Esta pesquisa foi financiada pela Fundação de Amparo à Pesquisa do Estado do Amazonas (FAPEAM) e Universidade do Estado do Amazonas (UEA).

\section{Colaborações:}

1 - concepção, projeto, análise e interpretação dos dados: Rosana Santos Brandão de Assis e Amélia Nunes Sicsú;

2 - redação do artigo e revisão crítica relevante do conteúdo intelectual: Ana Paula de Carvalho Portela, Pedro Fredemir Palha e Amélia Nunes Sicsú;

3 - aprovação final da versão a ser publicada: Lara Bezerra de Oliveira, Luana Cristina Fernandes Eufrasio, Ana Paula de Carvalho Portela, Pedro Fredemir Palha e Amélia Nunes Sicsú.

\section{Referências}

1. Raviglione M, Sulis G. Tuberculosis 2015: burden, challenges and strategy for control and elimination. Infect Dis Rep. 2016 Jun;8(2):6570. DOI: 10.4081/ idr.2016.6570

2. Fiseha D, Demissie M. Assessment of Directly Observed Therapy (DOT) following tuberculosis regimen change in Addis Ababa, Ethiopia: a qualitative study. BMC Infect Dis. 2015 Sep;30(15):405. DOI: 10.1186/s12879-015-1142-2

3. Brasil. Ministério da Saúde. Perspectivas brasileiras para o fim da tuberculose como problema de saúde pública. Boletim Epidemiológico [Internet]. 2016 [cited 2016 Oct 23];47(13). Available from: http://portalsaude.saude.gov.br/images/pdf/2016/ marco/24/2016-009-Tuberculose-001.pdf

4. Montagna MT, Napoli C, Tafuri S, Agodi A, Auxilia F, Casini B, et al. Knowledge about tuberculosis among undergraduate health care students in 15 Italian universities: a cross-sectional study. BMC Public Health. 2014;14(1):970. DOI:10.1186/1471-2458-14-970

5. Carvalho CF, Ponce MAZ, Silva-Sobrinho RA, Mendez RDR, Santos MA, Santos EM, et al. Tuberculose: conhecimento entre alunos de graduação em enfermagem. Rev Bras Enferm. 2019 Out;72(5):1279-87. DOI: 10.1590/0034-7167-2018-0384

6. Rocha MMS, Mocheuti KN, Silvestre GCSB, Lima CM, Ribeiro ADN. Sistematização da assistência de enfermagem na perspectiva do docente. J Health NPEPS [Internet]. 2019 [cited 2019 Oct 21];4(1):14452. Available from: https://pesquisa.bvsalud.org/ portal/resource/pt/biblio-999661

7. Guareschi APDF, Kurcgant P. Influência da formação docente no perfil do egresso de graduação em enfermagem. Cogitare Enferm [Internet]. $2014 \mathrm{jan} / \mathrm{mar}$ [cited 2016 Oct 25];19(1):101-8. Available from: http://revistas.ufpr. br/cogitare/article/view/35965

8. Brasil. Ministério da Saúde. Conselho Nacional de Saúde. Resolução n. 466, de 12 de dezembro de 2012. Aprova as diretrizes e normas regulamentadoras de pesquisas envolvendo seres humanos [Internet]. Brasília; 2012. (cited 2019 Oct 20]. Available from: https://bvsms.saude.gov. br/bvs/saudelegis/cns/2013/res0466_12_12_2012. html

9. Orlandi EP. Análise de discurso: princípios e procedimentos. 12a ed. São Paulo: Pontes; 2015.

10. Dornelas CC, Assolini FEP. A discursivização do feminino e suas relações com a docência: memória e atravessamentos discursivos. Entremeios: rev estudos disc. 2016;12:45-64. DOI: 10.20337/ ISSN2179-3514revistaENTREMEIOSvol12pagin a45a64

11. World Health Organization. Global Tuberculosis Report (2015) [Internet]. Geneva; 2016 [cited 2016 Oct 24]. Available from: http://www.who.int/tb/ publications/global_report/en/

12. Jung BC, Zillmer JGV, Cunha FTS, Gonzales RIC. Significados das experiências corporais de pessoas com tuberculose pulmonar: a construção de uma nova identidade. Texto contexto - enferm. 2018;27(2):e2030016. DOI: 10.1590/0104-070720180002030016

13. Tadesse S. Stigma against Tuberculosis Patients in Addis Ababa, Ethiopia. PloS One. 2016 
Apr;11(4):e0152900. DOI: 10.1371/journal. pone. 0152900

14. Grecco R, Oliveira CBB, Silva LMC, Souza KMJ, Santos GP, Palha PF. Directly observed treatment of tuberculosis: learning process in a higher education institution. Rev enferm UERJ [Internet]. $2014 \mathrm{jan} / \mathrm{fev}$ [cited 2016 Oct 25];22(1):77-82. Available from: http://www.facenf.uerj.br/v22n1/ v22n1a12.pdf

15. Daniel HB, Sandri JVA, Grillo LP. Implantação de política de educação permanente em saúde no Rio Grande do Sul. Trab educ saúde [Internet]. 2014 dez [cited 2019 Oct 21];12[3]:541-62. Available from http://www.scielo.br/scielo.php?script=sci_ arttext\&pid $=$ S1981-77462014000300541\&lng=en\& nrm $=$ iso

16. Temoteo RCA, Carvalho JBL, Lira ALBC, Lima MA, Sousa YG. Enfermagem na adesão ao tratamento da tuberculose e das tecnologias da saúde no contexto da atenção básica. Esc Anna Nery. 2019;23(3):e20180321. DOI: 10.1590/2177-9465-ean-2018-0321

17. Ruffino Netto A. Tuberculose: a calamidade negligenciada. Rev Soc Bras Med Tropical. 2002;35(1):51-8. DOI: 10.1590/S0037-86822002000100010

18. Carvalho VL, Oliveira ALC, Alves IKS, Silva RL, Silva CB. Competências para promoção da saúde em formandos dos cursos da área da saúde. Rev enferm UFPE on line. 2017 ago;11(Suppl 8):3269-78 DOI: 10.5205/reuol.11135-99435-1-ED.1108sup201711

19. Peruhype RC, Acosta LMW, Ruffino Netto A, Oliveira MMC, Palha PF. Distribuição da tuberculose em Porto Alegre: análise da magnitude e coinfecção tuberculose-HIV. Rev esc enferm USP [Internet]. 2014 Dec [cited 2019 Oct 21];48(6):1035-43. Available from: http://www. scielo.br/scielo.php?script=sci_arttext $\&$ pid $=$ S008062342014000601035\&lng=en

20. Ceccon RF, Maffacciolli R, Burille A, Meneghel SN, Oliveira DLLC, Gerhardt GE. Mortalidade por tuberculose nas capitais brasileiras, 20082010. Epidemiol Serv Saúde [Internet]. 2017 June [cited 2019 Oct 21];26(2):349-58. Available from: http://www.scielo.br/scielo.php?script=sci_ arttext\&pid=S2237-96222017000200349\&lng=en

21. Saunders MJ, Carlton AE. Fighting poverty to prevent tuberculosis. Lancet Infect Dis. 2016 Apr;16(4):395-6. DOI: 10.1016/S1473-3099(15) 00434-X

22. Cavalcante EFO, Silva DMGV. O compromisso do enfermeiro com o cuidado à pessoa com tuberculose. Texto Contexto-enferm. 2016;25(3): e3930015. DOI: 10.1590/0104-07072016003930015

23. Brasil. Ministério da Saúde. Secretaria de Vigilância em Saúde. Departamento de Vigilância das Doenças Transmissíveis. Manual de Recomendações para o Controle da Tuberculose no Brasil. Brasília; 2019.

24. Costa MR, Queiroz RCS, Rocha TAH, Silva NC, VissociJRN, Tonello AS, et al. Characteristics of basic health units and detection of tuberculosis cases. Rev Soc Bras Med Trop. 2019 Jan;52:e-20180230. DOI: $10.1590 / 0037-8682-0230-2018$

25. Silva Sobrinho RA, Souza AL, Wysocki AD, Silva LMC, Beraldo AA, Villa TCS. Conhecimento de Enfermeiros de Unidades de Atenção Básica acerca da Tuberculose. Cogitare Enferm [Internet]. 2014 [cited 2016 Nov 4];19(1):34-40. Disponível em: https://revistas.ufpr.br/cogitare/article/ view/35930/22161

Recebido: 23 de março de 2019 Aprovado: 30 de outubro de 2019

Publicado: 29 de janeiro de 2020

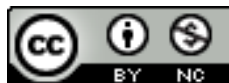

A Revista Baiana de Enfermagem utiliza a Licença Creative Commons - Atribuição-NãoComercial 4.0 Internacional. https://creativecommons.org/licenses/by-nc/4.0/ Este artigo é de acesso aberto distribuído sob os termos da Licença Creative Commons (CC BY-NC). Esta licença permite que outros remixem, adaptem e criem a partir do seu trabalho para fins não comerciais. Embora os novos trabalhos tenham de lhe atribuir o devido crédito e não possam ser usados para fins comerciais, os usuários não têm de licenciar esses trabalhos derivados sob os mesmos termos. 